\title{
Perforación de colon secundaria a empalamiento autoinfligido: a propósito de un caso
}

\author{
Colon perforation secondary to self-inflicted impalement: a case report
}

\author{
Iván Lozada-Martínez" *, Álvaro Periñan-Pérez', Gian Núñez-Rojas ${ }^{1,2}$, María Bolaño-Romero ${ }^{1}$ y \\ Willfrant Muñoz-Murillo²

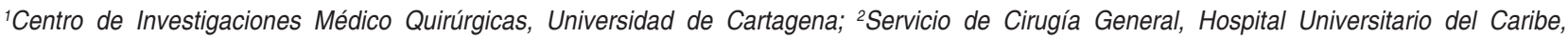 \\ Universidad de Cartagena. Cartagena, Colombia
}

\section{Resumen}

El empalamiento es una causa grave y rara de lesión anorrectal, pudiendo darse en multitud de contextos, ya sea de índole accidental, psiquiátrica o por prácticas sexuales o criminales. El objetivo de este manuscrito es mostrar al autoempalamiento como agente causante de lesión anorrectal, de presentación extraordinaria. Se presenta un caso de empalamiento autoinfligido con perforación intestinal en un hombre de 44 años, que acudió al servicio de urgencias sin el objeto causante de la lesión y con un cuadro de abdomen agudo, debido a hemoperitoneo, peritonitis fecal y perforación en la unión rectosigmoidea.

Palabras clave: Conducta autodestructiva. Estreñimiento. Perforación intestinal. Peritonitis.

\begin{abstract}
The impalement is a severe and rare cause of the anorectal lesion and can occur in a multitude of contexts, whether accidental, psychiatric or due to sexual or criminal practices. The objective of this manuscript is to show self-packing as the causative agent of anorectal lesion, being of extraordinary presentation. We present the case of self-inflicted impalement with intestinal perforation in a 44-year-old man, who attends the emergency department without the cause of the injury and with an acute abdomen, due to hemoperitoneum, fecal peritonitis and a perforation in the rectosigmoid junction.
\end{abstract}

Key words: Self-injurious behavior. Constipation. Intestinal perforation. Peritonitis.

\section{Introducción}

El empalamiento es una causa excepcional del trauma anorrectal'1. La lesión por empalamiento es un tipo específico de trauma definido como una herida penetrante causada por un objeto con punta roma, relacionada principalmente con caídas, actividades sexuales peligrosas o deslizamiento con una fuerza externa fuerte $^{2}$. La grave naturaleza de tales incidentes se demuestra en series de casos reportadas con unas tasas de mortalidad del $20-50 \%{ }^{3}$. Estas estadísticas generalmente se asocian con complicaciones de lesiones por caída, contaminación de heridas y lesiones en órganos profundos, lo que culmina en hemorragia, disfunción orgánica e infección ${ }^{2,4}$. Por ello, cualquiera que sea la causa del empalamiento, el diagnóstico

\footnotetext{
Correspondencia:

*Iván Lozada-Martínez

Las Gaviotas, 1

Fecha de recepción: 02-03-2020

C.P. 130004, Cartagena de Indias, Colombia

E-mail: ivandavidloma@gmail.com

0009-7411/@ 2020 Academia Mexicana de Cirugía. Publicado por Permanyer. Este es un artículo open access bajo la licencia CC BY-NC-ND (http://creativecommons.org/licenses/by-nc-nd/4.0/).

Cir Cir. 2020;88(S2):31-34 Contents available at PubMed www.cirugiaycirujanos.com
} 
preciso de las lesiones asociadas no es fácil y la ausencia de una herida externa superficial del margen anal no refleja la verdadera gravedad del daño". El objetivo de este artículo es exponer al autoempalamiento como agente causante de lesión anorrectal, que es de presentación extraordinaria y se encuentran muy pocos casos registrados en la literatura.

\section{Caso clínico}

Varón de 44 años que acude a la urgencia de la E.S.E. Hospital Universitario del Caribe por un cuadro clínico de 18 horas de evolución, consistente en dolor abdominal tipo cólico asociado a dificultad para la micción y tenesmo rectal. El paciente refirió que en la madrugada sintió la necesidad de defecar y, al no conseguirlo, se introdujo por el ano un cuerpo extraño identificado como un trozo de madera o «palo» envuelto en plástico para de este modo lograr la defecación, retirándolo inmediatamente. Como antecedentes relevantes refirió consumo de cocaína (informó no estar drogado en el momento del acto). En la exploración física se evidencia abdomen blando, doloroso a la palpación generalizada, de predomino en flancos bilaterales, sin masas ni megalias, y sin signos de irritación, pero con presencia de defensa voluntaria. Se le realiza una radiografía abdominal que evidencia ausencia de aire distal (imagen sugestiva de neumoperitoneo) (Fig. 1), y una ecografía abdominal que revela líquido libre particulado, por lo que se decidió realizar cirugía laparoscópica exploratoria.

Durante la intervención se evidencia hemoperitoneo $\left(1000 \mathrm{~cm}^{3}\right)$ con peritonitis fecal de cuatro cuadrantes, realizándose drenado de colección peritoneal. En la revisión sistemática de la cavidad se observa una perforación de la unión rectosigmoidea de $6 \times 4 \mathrm{~cm}$ con bordes desvitalizados y necróticos (Fig. 2), por lo que se hace una resección y se lleva a cabo colostomía izquierda en Hartmann con colocación de bolsa, sin complicaciones. En el posquirúrgico se instauró tratamiento con dipirona y ampicilina-sulbactam. El paciente evoluciona de forma satisfactoria y se da de alta a los 5 días de haber sido intervenido.

\section{Discusión}

Las lesiones por empalamiento son causadas por la penetración de un objeto de manera directa al cuerpo, pudiendo este ser retenido por estructuras internas, lo que constituye una emergencia su extracción forzosa por fuera del ámbito hospitalario ${ }^{5}$. La evaluación de la

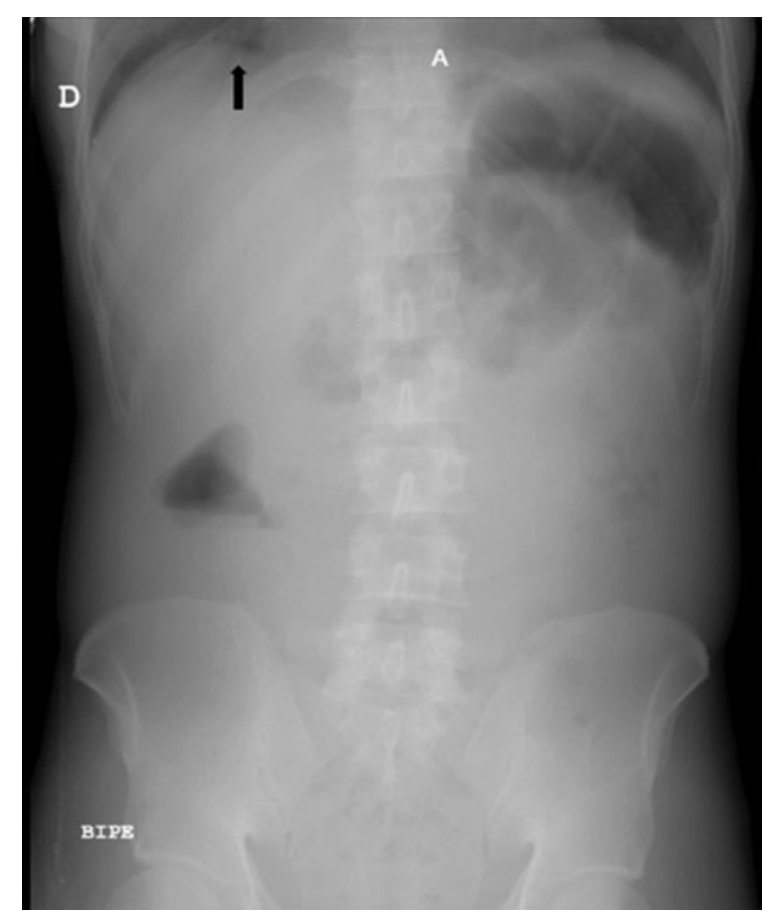

Figura 1. Radiografía de abdomen en la que se evidencia la ausencia de aire distal, lo que sugiere neumoperitoneo.

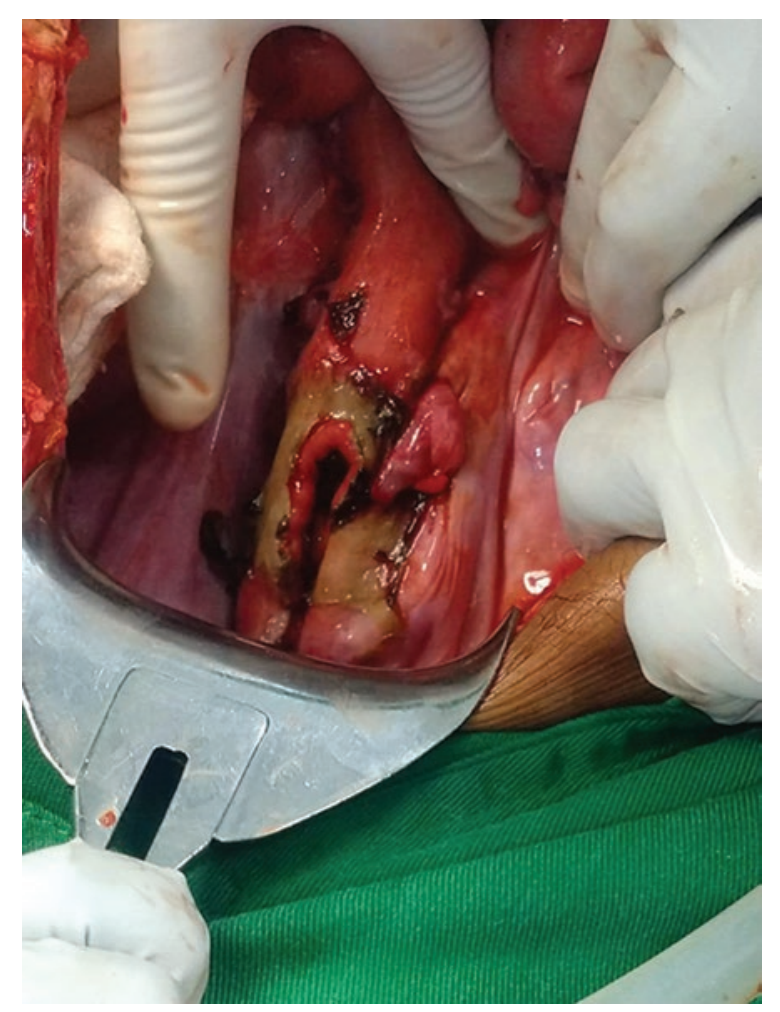

Figura 2. Fotografía en la que se observa la perforación de la unión rectosigmoidea con bordes desvitalizados y necróticos.

sospecha de lesión por empalamiento rectal implica una anamnesis detallada y una exploración física 
minuciosa, en la que se deben identificar el objeto, la fuerza y la dirección de penetración. Del mismo modo, el hecho de que un paciente refiera síntomas gastrointestinales, se torne miedoso y angustiado, se niegue a estar en posición de sedestación y trate de tergiversar el interrogatorio eleva considerablemente la creencia de que haya sufrido un trauma anorrectal. En la exploración física se debe realizar de forma completa la revisión del abdomen, la inspección del periné, un examen rectal digital y, en las mujeres, un examen vaginal. A pesar de lo anterior, la falta de hallazgos físicos no descarta la lesión intraabdominal ${ }^{6}$. La radiografía simple de abdomen puede ayudar a determinar si existe perforación intestinal por la presencia de aire o líquido en la cavidad abdominal ${ }^{7}$. Las recomendaciones generales para el manejo de las lesiones por empalamiento se basan en que se debe evitar la manipulación o eliminación del objeto empalado; esto podría volver a arrendar el efecto de taponamiento que ejerce el objeto en los vasos sanguíneos, resultando en una hemorragia masiva ${ }^{8}$, así como también la posibilidad de generar astillas o roturas del objeto. Idealmente se debe cortar el objeto para facilitar la movilización o posicionamiento. Todo objeto empalado debe considerarse altamente contaminado, y por lo tanto, debe administrarse cobertura antibiótica de amplio espectro y antitetánica ${ }^{9}$. De forma esquemática, el abordaje del empalamiento puede realizarse según diversas clasificaciones de lesiones anorrectales. En niños, Black, et al. ${ }^{10}$ describieron que para las lesiones tempranas admitidas se propone la reparación primaria sin colostomía en el grado I (lesión superficial del canal anal o de la membrana mucosa rectal) y el grado II (lesión de espesor total por debajo del esfínter anal interno o externo). La colostomía es necesaria en las lesiones de grado III (lesión de espesor total por encima del esfínter anal interno sin afectación peritoneal), IV (afectación peritoneal, pero sin lesión de otros órganos intraperitoneales) y $\mathrm{V}$ (lesión asociada a otros órganos intraperitoneales) ${ }^{1,11}$.

La cirugía para la corrección de la lesión generalmente se realiza a través de laparoscopía, puesto que se cree que es óptima para identificar la ubicación precisa de la perforación ya que permite una excelente visualización de lesiones colorrectales intraperitoneales, logrando un lavado peritoneal rápido y una pronta reparación del daño ${ }^{11-13}$. Además, esta técnica tiene otras ventajas, tales como una rápida recuperación posoperatoria, una fácil movilización del paciente fuera de la cama y una estancia hospitalaria más corta $^{14,15}$. Por lo tanto, se puede decir que la intervención laparoscópica es el tratamiento de elección.
En contraste, nuestro paciente asistió al servicio de urgencias sin signos de inestabilidad hemodinámica a pesar del hallazgo de un hemoperitoneo de $1000 \mathrm{~cm}^{3}$, muy probablemente resultado de haber retirado el objeto empalado; este hecho también puede explicar la peritonitis fecal en cuatro cuadrantes observada durante la cirugía laparoscópica. Hay que considerar que el paciente no presentó lesiones aparentes en la exploración física, lo que refleja que la falta de estas no se traduce en ausencia de daño intraabdominal. Se realizó laparotomía exploratoria junto con la colostomía, teniendo en cuenta el grado de la lesión encontrada (bordes desvitalizados y necróticos). Durante el posoperatorio se cumplió con el esquema antibiótico de amplio espectro en concordancia con las recomendaciones dictadas por la literatura. El análisis de este caso es un claro ejemplo de las advertencias y las consecuencias de determinadas acciones, así como de las medidas quirúrgicas dependiendo de la lesión.

\section{Conclusión}

El autoempalamiento es una causa singular de trauma anorrectal, y más aún en el contexto de este paciente, cuyo argumento fue el estar estreñido. El trauma anorrectal puede tener una elevada mortalidad, por lo que es necesario hacer un diagnóstico oportuno y establecer medidas pertinentes para minimizar riesgos, como evitar el retiro del objeto empalado y la terapia profiláctica con antibióticos de amplio espectro. Una buena anamnesis y una exploración asertiva ayudan enormemente en el abordaje de este tipo de trauma, en especial cuando el paciente trata de ocultar el caso por temor a señalamientos. Siempre se debe tener presente que la ausencia de lesiones aparentes no descarta afectación intraabdominal.

\section{Conflicto de intereses}

Los autores declaran que no existe ningún conflicto de intereses.

\section{Financiamiento}

Los autores declaran que no recibieron patrocinio para llevar a cabo este artículo.

\section{Responsabilidades éticas}

Protección de personas y animales. Los autores declaran que los procedimientos seguidos se 
conformaron a las normas éticas del comité de experimentación humana responsable y de acuerdo con la Asociación Médica Mundial y la Declaración de Helsinki.

Confidencialidad de los datos. Los autores declaran que han seguido los protocolos de su centro de trabajo sobre la publicación de datos de pacientes.

Derecho a la privacidad y consentimiento informado. Los autores han obtenido el consentimiento informado de los pacientes y/o sujetos referidos en el artículo. Este documento obra en poder del autor de correspondencia.

\section{Bibliografía}

1. Akakpo-Numado G, Boume M, Mihluedo-Agbolan K, Simlawo K Adabra K, Tekou H. Perforation of large and small intestines by impalement in a 13-year-old boy. J Ped Surg Case Report. 2014;2:206-8.

2. Oya S, Miyata K, Yuasa N, Takeuchi E, Goto Y, Miyake H, et al. Impalement injury to the left buttock with massive bleeding: a case report. Nagoya J Med Sci. 2013;75:147-52.
3. Klein RR, Scarborough RA. Traumatic perforations of the rectum and distal colon. Am J Surg. 1953;86:515-22.

4. Bemelman M, Hammacher E. Rectal impalement by pirate ship: a case report. Injury Extra. 2005;36:508-10.

5. Mohan R, Ram DU, Baba YS, Shetty A, Bhandary S. Transabdominal impalement: absence of visceral or vascular injury a rare possibility. $\mathrm{J}$ Emerg Med. 2011;41:495-8.

6. Kim S, Linden B, Cendron M, Puder M. Pediatric anorectal impalement with bladder rupture: case report and review of the literature. J Pediatr Surg. 2006;41: E1-3.

7. Hsieh MJ, Lu TC, Chen SY, Chen SC. Pneumoretroperitoneum as the manifestation of rectal perforation. J Emerg Med. 2011;41:536-7.

8. Lupaşcu C, Fotea V, Sârbu P, Andronic D. Rectal impalement injury: from cruelty to salvage endeavour. Chirurgia. 2015;110:60-5.

9. Rosat A, Sánchez JM, Chocarro C, Barrera M. Impalement injury by glass shard with delayed colonic perforation. Pan Afr Med J. 2015;21:330.

10. Black T, Pokorny W, McGill C, Harberg F. Ano-rectal trauma in children. J Pediatr Surg. 1982;17:501-4.

11. Carlos B, Newton J, Cecilia D, Heladio M, Héctor S. Perforación traumática del conducto anorrectal. Cir Cir. 2005;73:453-6.

12. Bonnard A, Zamakhshary M, Wales PW. Outcomes and management of rectal injuries in children. Pediatr Surg Int. 2007;23:1071-6.

13. Travassos DV, Chrzan R, van der Zee D. Late laparoscopic management of traumatic rectal injury without protective colostomy. J Laparoendosc Adv Surg Tech A. 2009;19:843-4.

14. Marwan A, Hamon CM, Georgeson KE, Smith GF, Muensterer OJ. Use of laparoscopy in the management of pediatric abdominal trauma. J Trauma. 2010;69:761-4.

15. Boettcher M, Kanellos-Becker I, Wenke K, Krebs TF. Rectum perforation after broomstick impalement in a 17-year-old: case report and review. Pediatr Emerg Care. 2013;29:510-2. 